\title{
Meru University of Science and Technology Research Ethics Review System: A SWOT Analysis
}

\author{
Joses Muthuri Kirigia, Rosenabi Deborah Karimi Muthuri, Newton Gitonga \\ African Sustainable Development Research Consortium (ASDRC), Nairobi, Kenya \\ Email: kirigiajoses@gmail.com
}

How to cite this paper: Kirigia, J.M., Muthuri, R.D.K. and Gitonga, N. (2018) Meru University of Science and Technology Research Ethics Review System: A SWOT Analysis. Journal of Biosciences and Medicines, 6, 26-44.

https://doi.org/10.4236/jbm.2018.612003

Received: October 23, 2018

Accepted: December 2, 2018

Published: December 5, 2018

Copyright (c) 2018 by authors and Scientific Research Publishing Inc. This work is licensed under the Creative Commons Attribution International License (CC BY 4.0).

http://creativecommons.org/licenses/by/4.0/

\begin{abstract}
The objectives of this article are as follows: 1) to propose a university research ethics system framework, 2) to provide a brief anatomy of the Meru University of Science and Technology (MUST) Institutional Research Ethics Review Committee (MIRERC), 3) to perform a SWOT (Strengths, Weaknesses, Opportunities, and Threats) analysis of MIRERC, and 4) to make recommendations for improving its performance. The 13-member multi-disciplinary MIRERC was established in April 2017 to provide effective ethical oversight of research undertaken by the University's scholarly community. Strengths of the MUST research ethics review system include a functional MIRERC, a pertinent national law and ethical guidelines, an Innovation and Enterprise Centre that could house a dedicated MIRERC Secretariat, and a supportive University Management Board. The weaknesses include lack of graduate schools to assure scientific rigor of proposals before submission to the MIRERC, lack of research ethics training in most school's curricula, absence of a dedicated MIRERC Secretariat, undergraduate research proposals being not ethically reviewed, dearth of faculty trained in research ethics, and lack of an operating budget for MIRERC work. The opportunities include existence of about 22 accredited Institutional Research Ethics Review Committees (IRERC) in Kenya, existence of international standards and operational guidance for ethics review, availability of guidelines and codes of best ethical practices in research, existence of a free automated platform called Research for Health Innovation Organizer (RHInnO) Ethics for managing the ethics review process, and availability of external resources for strengthening IRERCs. In order to improve the performance and sustainability of the MUST research ethics system, there is need to include research ethics training in all undergraduate and post-graduate curricula, create a dynamic database of potential research ethics reviewers, allocate a percentage of the annual MUST research budget for MIRERC operations, charge a graduated fee for proposal ethics review, re-
\end{abstract}


quire all students' and faculties' internal and external research proposals be cleared by the MIRERC, and use the RHInnO Ethics platform to manage the ethics review process.

\section{Keywords}

Meru University of Science and Technology, Kenya, Institutional Research Ethics Review Committee, Research Ethics System, SWOTs Analysis

\section{Introduction}

The Meru University of Science and Technology (MUST) is a public university located in Nchiru, 15 kilometres from Meru Town, Meru County, Kenya. The University started in 1979 as the Meru College of Technology; and through Legal Notice No. 103, on $18^{\text {th }}$ July, 2008, it became the Meru University College of Science and Technology, a constituent college of the Jomo Kenyatta University of Agriculture and Technology (JKUAT) [1]. On $1^{\text {st }}$ March, 2013, Kenya Government issued the MUST Charter, which approved its status as a fully-pledged public university [2].

Three of the 11 objectives of university education in Kenya are related to research excellence. These include advancement of knowledge through teaching, scholarly research, and scientific investigation; promotion of the highest standards in, and quality of, teaching and research; and dissemination of the outcomes of research conducted by the university to the general community [3].

One of the six objectives of the MUST is to establish sustainable research initiatives that promote societal development [2]. Two of the 15 MUST functions are to:

"provide excellent teaching, training, scholarship, entrepreneurship, innovation, research, consultancy and extension services with emphasis on basic and applied research (Article 6c); provide programmes, products and services in ways that reflect proper ethics founded in the principles of equity and social justice (Article 61)" [2].

The MUST envisions become a world-class university of excellence in science, technology, and innovation. Its mission is to provide quality education, training, and research in science, technology, and innovation, to produce globally competitive graduates [2].

The University has eight schools, namely, the School of Agriculture and Food Science (SAFS), School of Business and Economics (SBE), School of Computing and Information (SCI), School of Education (SEd), School of Engineering and Architecture (SEA), School of Health Sciences (SHS), School of Nursing (SoN), and School of Pure and Applied Sciences (SPAS) [1]. The total student population in 2017 was 5471. This comprised 4991 (91.2\%) undergraduates, 138 (2.5\%) pursuing a Master's degree, $16(0.3 \%)$ pursuing a Doctorate in Philosophy, 255 (4.7\%) pursuing a diploma, and $71(1.3 \%)$ pursuing a certificate [4]. In some of 
the schools, for example SHS, fourth year undergraduate students are obliged to design small-scale studies and submit a research report before graduation. The Commission for University Education (CUE) requires Master's and PhD students to conduct original research and submit a dissertation/thesis in partial fulfilment of their degree (Standard PROG/STD/02) [5]. Thus, the undergraduate, Master's and $\mathrm{PhD}$ research projects have ethical implications for human and non-human research subjects.

The Kenya National Council for Science and Technology (NCST) requires all research projects that involve human beings as research participants to satisfy the appropriate international ethical standards for research [6]. It also requires that all those applying for a research permit to provide a letter of ethics approval from recognized ethical research committees [7]. Thus, it is vital for a university that aspires to become a world-class university of excellence in science, technology, and innovation to have an institutional ethics review system. The MUST Research Ethics System as explained in section 2 contributes to safeguarding the well-being of all potential and actual research participants.

The objectives of this article are as follows: 1) to propose a university research ethics system framework, 2) to provide a brief anatomy of the MUST Institutional Research Ethics Review Committee (MIRERC), 3) to perform a SWOT (Strengths, Weaknesses, Opportunities, and Threats) analysis of the MIRERC, and 4) to make recommendations for improving its performance.

\section{MUST Research Ethics System}

\subsection{Research Ethics System Framework}

The MUST research ethics review system (MRERS) can be defined as people and institutions whose primary purpose is to regulate, in line with international ethical guidelines, research conducted by students, staff and collaborators, with a view to safeguarding the dignity, rights, safety, and well-being of all actual or potential human and non-human research participants or subjects. As depicted in Figure 1, the MRERS goals are 1) to create requisite university-wide capacities in ethical conduct of research; and 2) to protect and promote well-being of all actual and potential human and non-human research participants or subjects. The MRERS has four functions: research ethics governance; developing and sustaining research ethics resources; financing of MUST Institutional Research Ethics Review Committee (MIRERC) operations; and provision of ethical services.

The function of research ethics governance has three components. First, MRERS organizational structure, which includes the MUST Research and Publication Committee that administers the MUST Research Fund; Departmental Post-Graduate Committees, which review scientific rigor of student research proposals; and the MUST Institutional Research Ethics Review Committee (MIRERC) that provides ethical oversight of all research conducted by MUST scholarly community. Second, development of standard operating procedures 
and tools (forms and questionnaires) for MIRERC. Third, development of strategic and annual action plans for MIRERC. Fourth, monitoring and evaluating implementation of MIRERC annual action plans, which would also include monitoring of the implementation of approved research studies.

The function of developing and sustaining research ethics resources has three components. First, human resources for research ethics, which includes MIRERC Secretariat, lecturers and students trained in international ethical standards and guidelines, and the MIRERC standard operating procedures and tools. Second, the MIRERC physical infrastructure, including offices for the Secretariat, conference room for meetings, filing cabinets, computers (with internet and email connectivity), printers, and LCD projector(s). Third, consumable materials, e.g. printer cartridges, stationery.

The function of financing of MRERS has two components. First, raising adequate funding for covering capital and recurrent costs of operating the MRERS. The MRERS could potentially be allotted a percentage of the annually allocated MUST Research Fund (MRF); and MIRERC could charge graduated fees for review of MRF research proposals, externally funded proposals, and doctor of philosophy and master's degree proposals. Second, ensuring efficient (allocation and use without waste) and accountable use of all MRERS funds.

\section{MUST RESEARCH ETHICS REVIEW SYSTEM (MRERS) GOALS}

1). The creation of university-wide capacities for ethical conduct of research.

2). The protection and promotion of well-being of all actual and potential human and non-human research participants or subjects.

RESEARCH ETHICS GOVERNANCE

a). MRERS organizational structure.

b). Development of standard operating procedures and tools for MIRERC.

c). Development of strategic and annual action plans for MIRERC.

d). Monitoring and evaluating implementation of MIRERC.

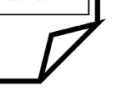

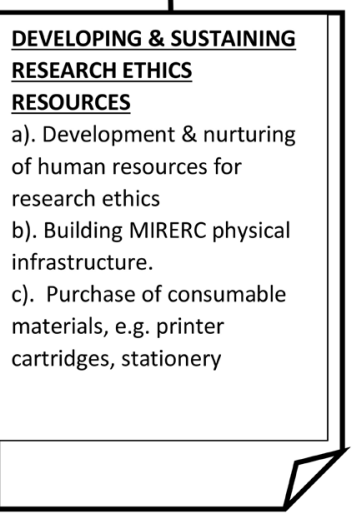

\section{FINANCING OF MRERS}

(a). Mobilization of funds from the MUST Research Fund (MRF); and charge graduated fees for review of MRF research proposals, externally funded proposals, and doctor of philosophy and master's degree proposals.

(b). Ensuring efficient and accountable allocation and use of all MRERS funds.

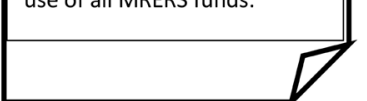

PROVISION OF ETHICAL SERVICES

a). Ethical review and approval of research internally and externally originating research proposals.

b). Investigation of reports of suspected adverse reaction.

c). Ethical audit of approved studies before project closure.

Figure 1. MUST Research Ethics Review System (MRERS). Source: Authors' drawing. 
The function of provision of ethical services has three components. First, ethical review and approval of research proposals originating from MUST. Second, ethical review and approval of research proposals originating from outside MUST. Third, investigation of reports of suspected adverse reaction among research subjects. Four, ethical audit of approved studies before project closure.

\subsection{A Brief Anatomy of the MIRERC}

In accordance with the Kenya NCST guidelines for ethical conduct of research involving human subjects in Kenya, in April 2017, the University established the MIRERC for ethics review of research proposals. The role of the MIRERC is to review research projects undertaken by members of staff, registered students, and affiliates of the University, to contribute to safeguarding the dignity, rights, safety, health, and well-being of all actual or potential human and non-human research participants. The Committee is mandated to provide independent, competent, and ethical review of research proposals by following the procedures outlined in the MUST Standard Operating Procedures (SOPs) of the MIRERC [8].

The Vice-Chancellor (VC) appointed 13 MIRERC members, initially for a period of three years (renewable once). The multi-disciplinary committee consists of three business administration specialists, a health economist, an engineer, a computer scientist, a clinical medicine specialist, a nursing scientist, an education specialist, a psychologist, a librarian, a biologist, and a lawyer. Six (46\%) of the members are female. Prior to joining the MUST, the health economist used to co-chair the World Health Organization (WHO) African Region Research Ethics Review Committee. The members were appointed in their personal capacities based on their scientific and/or ethical expertise, as well as their willingness to voluntarily review research protocols/proposals. Additional selection criteria for MIRERC members were multi-disciplinarity (representation of all MUST Schools areas of research focus), gender balance, and non-affiliation to sponsors / funders of research.

The MIRERC was constituted in nine steps. First, through the Deputy Vice-Chancellor Academic, Research and Student Affairs (DVC-ARS), the Director of Research (DOR) wrote a memorandum to the Vice-Chancellor (VC), seeking authorization to constitute an institutional research ethics committee. Second, the MUST Research, Printing and Publication Committee (RPPC) identified a multi-disciplinary and inter-school team of potential MIRERC members.

Then, through the DVC-ARS, the DOR wrote a memorandum to the VC, proposing MIRERC membership and requesting approval and appointment, after which the VC approved the all the proposed members and wrote memorandums to nominated staff, appointing them as MIRERC members.

In the next step, the DOR organized a one-day ethics training for MIRERC members and shared various international research ethics guidelines. The ethical guidelines and documentation shared included international ethical guidelines 
for health-related research involving humans [9], a medical ethics manual [10], guidelines for ethical conduct in the care and use of nonhuman animals in research [11], a guide for research ethics committee members [12], documents of research ethics committees on basic concepts for capacity-building [13], operational guidelines for ethics committees that review biomedical research [14], a document good clinical practices [15], the Belmont report ethical principles and guidelines for the protection of human subjects of research [16], and the Declaration of Helsinki [17].

The training touched on the human development landscape in Africa; research ethics committee stakeholders; examples of major unethical events that have occurred in the past (e.g. the Nazi Medical War Crimes that led to the Nuremberg Code [18], the Thalidomide disaster that caused severe birth defects [19], the Tuskegee 1932 and 1972 study of untreated syphilis among 600 African American males [20], and the New Zealand study of women with untreated carcinoma in situ of the cervix [21]); ethical principles of respect for persons, beneficence, and justice; application of ethical principles through informed consent, risk, and benefit assessment, and fair procedures and outcomes in the selection of research participants; implication of unethical studies; international benchmarks for ethical research; collaborative partnership; CIOMS ethical guidelines for biomedical research; responsibilities of MIRERC members; MIRERC resources, training and independence; MIRERC operations; documentation of MIRERC decisions; elements of informed consent (competence, disclosure, comprehension, and voluntariness); who gives consent; vulnerable population groups; legally acceptable/authorised representative for vulnerable groups; basic elements of information sheet; and MIRERC operational tools.

In the next step, the DOR reviewed the ethics guidelines of various organizations and institutions, and proposed a draft of Standard Operating Procedures (SOPs) and 12 forms to the MIRERC. A meeting of the MIRERC reviewed, revised, and finalized the SOPs for use in reviewing all types (human, animal, and plant) of research protocols [8]. The MIRERC SOPs have the following subsections: glossary, abbreviations, acknowledgement, constitutive terms, role of the MIRERC, general attitude of the MIRERC, research evaluation policy, independence of the MIRERC, membership, terms of reference, Secretariat, functions and responsibilities, regular MIRERC meetings, conduct of MIRERC meetings, special meetings, participant informed consent document and the consent process, submission of a research protocol/proposal, types of review, post approval protocol amendments and continued review, communication of review decisions, follow-up, monitoring of research, appeal against committee decisions, responsibility of the principal investigator in the review process, documentation and archiving, and references. The 12 MIRERC ethics review forms are as follows: application form for ethics review, abstract form, funding proto$\mathrm{col} /$ proposal format, Masters of Science (MSc) and Doctor of Philosophy (PhD) research proposal template, informed consent form (ICF), ethics reviewer's 
comments form, checklist for reviewers, continuing review form; amendment request form, unanticipated events prompt reporting form, annual progress report form, and close of project report form.

Subsequently, the DVC-ARS apprised the Deans and Directors of the establishment of the MIRERC at a Deans Committee meeting. He requested them to share information with all staff and post-graduate students, and to inform them that all their research protocols require MIRERC approval prior to implementation.

Then, the MUST Information and Technology Department developed an email address and a webpage for the MIRERC following a request from the Chairman of the MIRERC.

Finally, the DOR wrote a memorandum, through the DVC-ARS, to the VC requesting him to send a dossier to the National Commission for Science, Technology and Innovation (NACOSTI), applying for accreditation of the MIRERC. The dossier consisted of an application letter addressed to the Director-General of the NACOSTI; list of members of the MIRERC (indicating names, gender and area of expertise); SOPs of the MIRERC; training of MIRERC members; and summary of MIRERC activities. The NCST accreditation guidelines provide ethics committees and their appointing authorities with the requirements for accreditation [22].

\section{Summary of Proposals Reviewed and Recurring Gaps}

Figure 2 presents a flow chart of the MIRERC research proposal review and approval process at MUST.

Between April 2017 and January 2018, the MIRERC reviewed a total of 36 proposals following closely the CIOMS ethical basis for decision-making, which includes scientific design and conduct of the study; risks and potential benefits; selection of study population and recruitment of research participants; inducements, financial benefits, and financial costs; protection of research participants' privacy and confidentiality; informed consent process; and community considerations. About $26(21 \mathrm{MSc}$ and $5 \mathrm{PhD})$ were submitted by post-graduate students (72\%), 9 (25\%) were supported by the MUST Research Fund, and 1 (3\%) was funded externally. Approximately 21 (58\%) proposals were on public health, 8 (22\%) on epidemiology, 3 (8\%) on food science, 2 (6\%) on agronomy, and 1 (3\%) each on business and health information technology. Thirty-five of the proposals were revised by authors to incorporate MIRERC suggestions, re-reviewed by MIRERC reviewers, and granted approval for implementation. At the time of writing this paper, one author had not resubmitted the revised proposal to the MIRERC for re-review.

The following recurring scientific gaps were found in the post-graduate research proposals: 1) lack of knowledge/information gaps in the research problem statement $(16 / 26)$; 2) justification did not reflect potential benefit to all stakeholders, e.g. policy makers and planners at national and county governments, 
1. Post-graduate student (or faculty) prepares a research proposal under supervision

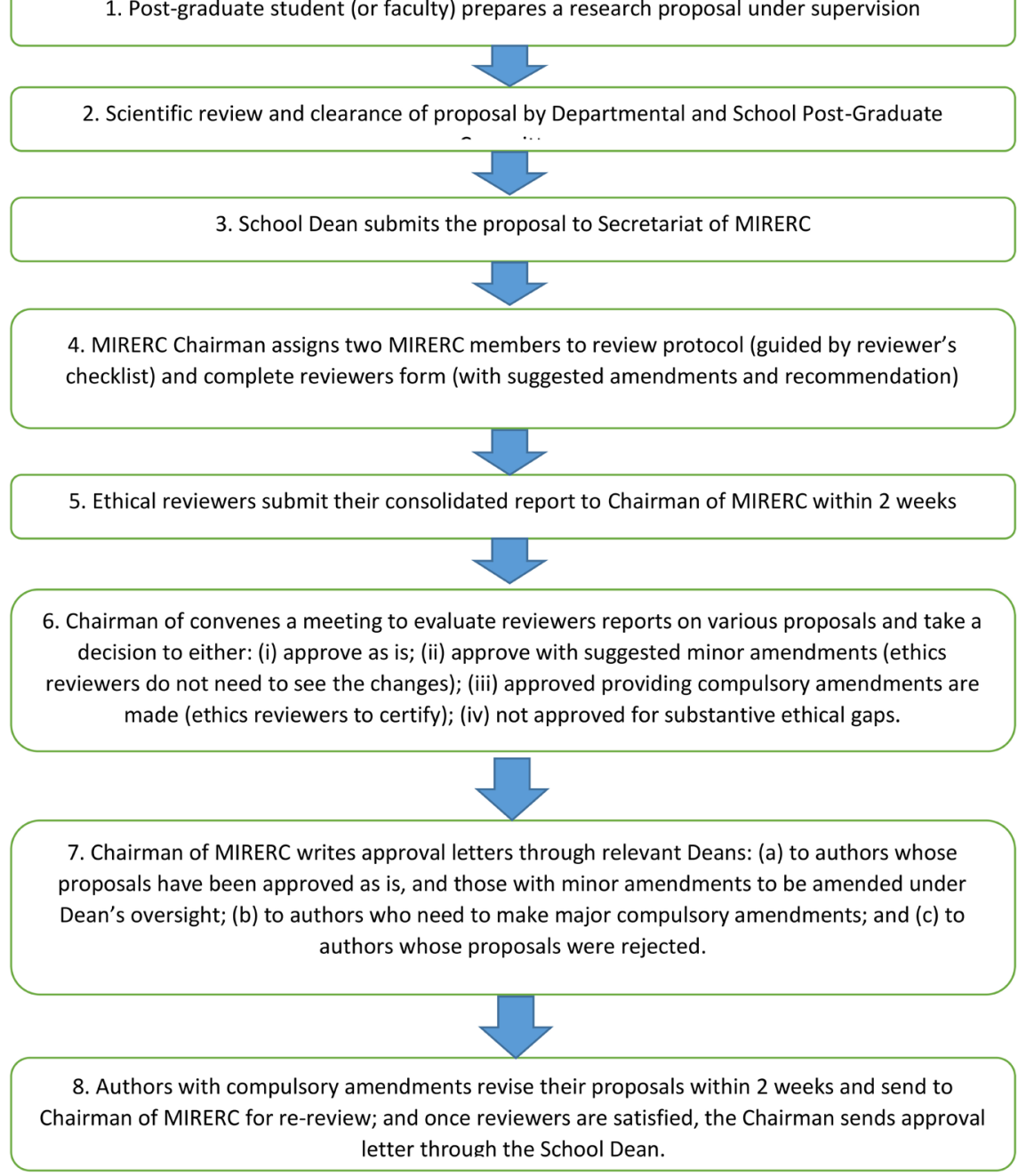

Figure 2. Flow chart of the MIRERC research proposal review and approval process at MUST. Source: Author's drawing.

programme manager's/service providers, local community (in which the study will be conducted), scholarly community, civil society organizations, and donors (9/26); 3) lack of (or inadequately formulated) conceptual framework, often not stating the individual dependent and independent variables, and models to be estimated (15/26); 4) no explanation of sampling technique(s) to be used to ensure equal probability (chance) of units in sample frame being drawn) (1/26); 5) data collection instruments either not appended or not referred to in the body of the proposal (1/26); 6) lack of uniform use of one style of referencing, e.g. the American Psychological Association, Harvard, or Vancouver style (2/26); and 7) no indication of whether scientific review was done by the School Departmental Graduate Committee (SDGC) prior to submission for ethical review and approval by MIRERC (16/26).

The frequently occurring ethical flaws included lack of explanation of how administrative permission would be obtained from the county government and 
institutions where the proposed study would be conducted (5/26); dearth of awareness of the MIRERC SOPs (1/26); complete omission of a section on ethical considerations (with exception of proposals from School of Health Sciences) (2/26); exclusion of information sheet and consent form, and when included, some elements of an ICF were missing (21/26); non-translation of ICF and data collection instruments into local languages (given that $22 \%$ of Kenyan adults are illiterate [23]) (26/26); and inadequate (or absence of) explanation of data management methods, including measures taken to ensure anonymity, confidentiality, and security of the personal information of research participants, who will have access to the data, and for how long data/samples will be stored for future use (23/26).

\section{Strengths of the MUST Research Ethics System}

This instrument in Appendix 1 was used to identify internal and external factors influencing the performance of the MUST research ethics system (MRES). There are five key strengths of the MUST research ethics review system. The first is the existence of a functional MIRERC with SOPs and relevant ethical forms [8]. Another strength is that MIRERC members have post-graduate expertise in their respective academic areas of specialization.

Third, the mandate and functions of the MIRERC are underpinned by strong national science, technology, and innovation laws and ethical guidelines. For example, Article 14 of the Science, Technology and Innovation Act No. 28 of 2013 requires that any person issued with a research permit must adhere to the code of ethics and guidelines [24].

The NCST, in accordance with Article III of the Science, Technology and Innovation Act, established the National Bioethics Committee (NBC) to spearhead the development of ethics committees in Kenya [24]. The NBC guidelines for accreditation of ethics review committees (REC) are meant to ensure that RECs have similar basic structures, capacities, and operational framework [22]. The guideline recognizes two types of institutional ethics review committees, the human ethics committee for protecting human research subjects and the animal ethics committee for safeguarding wellbeing of animal research subjects. The NCST guidelines for ethical conduct of biomedical research involving human subjects provides a background for the evolution of medical research ethics; a systematic and coherent framework for determining whether clinical research is ethical (value, scientific validity, fair subject selection, favorable risk-benefit ratio, independent review, informed consent, and respect for potential and enrolled subjects); general ethical principles (respect for persons, beneficence, and justice); 15 guidelines for protecting human subjects from harm; phases of clinical drug trials and vaccines; guidelines for externally sponsored research; and guidelines for constituting an ethical committee [7].

Fourth, the MUST UMB supports MIRERC work. Finally, the MUST has an Innovation and Enterprise Centre with underutilized office space, which could be used to house a dedicated MIRERC Secretariat. 


\section{Weaknesses of the MUST Research Ethics System}

There are a number of challenges confronting the MUST ethics review system. First, by $31^{\text {st }}$ January, 2018, the University did not have a graduate school to assure scientific rigor/veracity of protocols before submission to the MIRERC. As a stop gap measure, other schools in the University were instructed to establish Departmental Graduate Committees to review post-graduate students research proposals and propose allocation of supervisors to the DVC-ARS. However, there is still a need for a graduate school to coordinate the overall welfare of post-graduate students. In absence of a graduate school, MIRERC reviewers are also obliged to review the scientific aspects of the proposals/protocols.

Second, with exception of the School of Health Sciences, where undergraduate and graduate students receive basic training in research ethics, other schools do not have courses on research ethics. Therefore, in schools that do not provide research ethics training, awareness of the need for ethical clearance of non-medical research proposals is limited.

Third, the University does not have an office dedicated to the management of MIRERC ethics review work. In 2017, the DOR, consisting of only the Director and a casual clerk/secretary, functioned as a secretariat for the MIRERC. As the numbers of proposals submitted for ethics review increases, there will be a need for an adequately staffed office that is dedicated to MIRERC work, to ensure efficient operations.

Fourth, undergraduate research proposals are not reviewed by the MIRERC prior to implementation, which exposes the University to the potential risk of unethical research. This is because the MIRERC is relatively nascent and does not have requisite capacities to process very large numbers of undergraduate proposals.

Fifth, the country (and indeed the African Region [25] [26]) has limited number of research ethics trainers, and thus, it was challenging to find external trainers. The VC wrote to the WHO Regional Office for Africa requesting for technical support to train MIRERC members, but there was no response.

Finally, there are a number of operational issues, including the lack of an operating budget for MIRERC work; absence of SOPs on animal-based (non-human) research; limited (but growing) research ethics review experience; MIRERC members have substantive teaching workload in addition to their research ethics review responsibilities; MIRERC members are not remunerated for their work, and in medium and long-term, as numbers of proposals increase, this might be a problem; and many of the proposal authors take longer than the required two weeks to address the ethics reviewer's suggestions.

\section{Opportunities for the MUST Ethics System}

A number of opportunities are potentially available to the MUST ethics review system. First, there exist about 22 institutional ethics review committees (IRERC) in Kenya that are accredited with the NACOSTI [27]. The MIRERC 
could potentially enhance its capabilities by networking and collaborating with these older IRERCs.

Another opportunity is the existence of international standards and operational guidance for ethics review of research involving human [28] and non-human participants. For instance, WHO has a publication with basic concepts for use in the capacity-building of research ethics committees [13], and guidelines on ethical issues in public health surveillance [29]. American Psychological Association (APA) has guidelines for ethical conduct in the care and use of non-human animals in research, which were developed by the APA Committee on Animal Research and Ethics in 2010-11 [11]. The Australian code for the care and use of animals for scientific purposes details the responsibilities of investigators, animal carers, institutions, and animal ethics committees, and it describes processes for accountability [30].

The availability of guidelines and codes on best ethical practices in research offer another opportunity. For example, the Nuremberg Code [18], Declaration of Helsinki [17], International Ethical Guidelines for Biomedical Research Involving Human Subjects [9]; Ethical Considerations in Biomedical HIV Prevention Trials [31], United Nations Educational, Scientific and Cultural Organisation (UNESCO) Universal Declaration on Bioethics and Human Rights [32], and Ethics of Research related to Healthcare in Developing Countries [33].

Next is the accessibility of pertinent international statutes and regulations. For instance, the Universal Declaration of Human Rights [34]; International Council for Harmonisation of Technical Requirements for Pharmaceuticals for Human Use (ICH) Guideline for Good Clinical Practice [15]; ICH Guidelines on Choice of Control Groups and Related Issues in Clinical Trials [35]; Council of Europe Convention on Human Rights and Biomedicine [36]; Additional Protocol to the Convention on Human Rights and Biomedicine, concerning Biomedical Research [37]; Directive of the European Parliament and of the Council of 4 April 2001 on the approximation of the laws, regulations, and administrative provisions of the Member States relating to the implementation of good clinical practice in the conduct of clinical trials on medicinal products for human use [38]; and United States Food and Drug Administration regulations for the protection of human subjects [39].

Additionally, the Council on Health Research for Development (COHRED) has developed an automated platform called Research for Health Innovation Organiser (RHInnO Ethics), which facilitates and manages the entire research ethics review process [40]. RHInnO Ethics enables researchers to submit research protocol and supporting documents online, track the review and approval progress, receive automated notifications on the status, access ethics reviewers' reports on online, and access institutional templates and policy guidelines electronically [41]. It also facilitates reviewers to electronically receive and accept/decline invitations to review protocols, access assigned protocols and supporting documents, transmit review report to the administrator, and access 
available guidelines and policies for RECs [41]. RHInnO Ethics enables administrators to electronically receive notifications when new protocols are submitted, coordinate the beginning and end of a review cycle, conduct a quick internal appraisal of protocols before sending them to reviewers, invite reviewers, collate reviewers' comments and dispatch them to principal investigators, and monitor the progress of protocol review and send reminders in case of delays [41].

Finally, the availability of external resources for strengthening research ethics and regulatory bodies capacities (functionality and performance) is another opportunity. For example, the European and Developing Countries Clinical Trails Partnership (EDCTP) provides ethics grants to institutions in sub-Saharan Africa for establishment and strengthening of national ethics committees, institutional ethics review boards/committees, coordination, training, and networking [42]. Countries such as Benin, Botswana, Cameroon, Democratic Republic of Congo (DRC), Ethiopia, Gabon, Ghana, Kenya, Liberia, Malawi, Mozambique, Nigeria, Rwanda, Senegal, South Africa, Tanzania, Uganda, and Zimbabwe have received ethics grants in the past [43].

The John E. Fogarty International Center also supports research ethics capacity strengthening through two programmes, the International Research Ethics Education and Curriculum Development Award and International Bioethics Research Training Program [44].

\section{Threats to the MUST Ethics Review System}

There are five main threats to the MUST ethics review system. First, some students and staff, especially those conducting non-human research, might resist to submit their research proposals to the MIRERC for ethical review. Second, the Kenya National Research Fund (NRF) does not currently demand evidence of ethical approval of proposals submitted from national universities prior to funding commitment. Thus, the MUST scholarly community members competing for NRF may not have any incentive to submit their proposals to the MIRERC. Third, unless the MUST starts offering some honorarium to MIRERC reviewers, they might not consider continuing voluntary work in the medium and long-term. The potential conflict of interest among MIRERC members, who might review proposals from family members and close friends without declaring conflict of interest is another threat. Finally, the lack of formal linkages and collaboration between the MIRERC and other IRERCs in Kenya, in the African continent, and globally, might stifle members' continued research ethics learning.

\section{Recommendations}

The MUST research ethics system performance and sustainability can be improved in a number of ways. First, there is need for the UMB to include research ethics training in all undergraduate and post-graduate curricula. With requisite support from the UMB, the MIRERC could develop a programme for training of 
at least two research ethics trainers from each academic department. These trainers should be allocated requisite resources to enable them to train departmental faculty and students.

Second, the MIRERC should create a dynamic database of potential research ethics reviewers within Kenya. The Secretariat can implement this recommendation using the following approaches: 1) requesting for a list of bioethics experts from the Bioethics Society of Kenya [45] and 2) searching all academic and search engines for Kenyan authors of articles and books on research ethics [46].

Third, the UMB ought to allocate a percentage of the annual MUST Research Fund (MRF) budget to the MIRERC for use in paying honorariums to ethics reviewers of research proposals. The MUST currently pays Ksh 8000 (US\$ 80) to each internal and external scientific reviewer of MRF proposals. Thus, it is recommended that each ethics reviewer be paid the same honorarium per proposal reviewed.

Fourth, to further increase sustainability of the MIRERC, the UMB should consider charging a fee for ethics review. The nominal fee could be levied as a part of the tuition fees of students pursuing undergraduate-, master's- and doctoral programs. For example, the Moi University Institutional Research and Ethics Committee charges are as follows: Bachelors/Diploma: Ksh 1000 (US\$ 10), Master's: Ksh 2000 (US\$ 20), PhD: Ksh 3000 (US\$ 30), and Faculty/Post-Doctoral: Ksh 5000 (US\$ 50) per proposal [47].

Fifth, the UMB should institute a graduated ethics review charge for each externally funded research proposal towards the MIRERC kitty. For example, the fees for externally funded proposals at the Moi University Institutional Research and Ethics Committee are as follows: US $\$ 500$ for proposals worth less than US\$50,000, US\$ 1000 for those worth US\$50,000 - US\$100,000, and US\$ 1500 for proposals worth over US\$100,000 [47].

Sixth, the UMB ought to make it mandatory for all research proposals involving MUST students (undergraduate and post-graduate) to be subjected to MIRERC clearance prior to conduct of studies. Implementation of this recommendation presupposes the existence of an adequately staffed and financed the MIRERC. Otherwise, with its current meagre resource endowment, the MIRERC does not have the capacity to review all the undergraduate and post-graduate research proposals.

Seventh, the UMB should require all student and faculty research proposals prepared for submission to either the Kenya NRF or to international research funding organizations to be cleared by the MIRERC prior to their transmission for funding consideration.

Eighth, a revamped MIRERC should devise a mechanism for ensuring that highest ethical standards are upheld in the entire research cycle, including proposal development, implementation, dissemination, and closure.

Finally, the MIRERC Secretariat, Chairman, and membership should download the RHInnO Ethics platform, acquaint themselves with it, and start using it. If training is required, through the VC, the Chairman should request for tech- 
nical support from the COHRED. According to Mokgatla et al. [40], ethics review committees that have institutionalized RHInnO Ethics have realized remarkable benefits in terms of improved data security, submission process, communication, standardization, and cost savings.

\section{Conclusions}

This paper provides a brief anatomy (including steps followed in its formation) and a SWOTs analysis of the MUST research ethics system. Its strengths include a functional MIRERC, existence of pertinent national law and ethical guidelines, existence of an Innovation and Enterprise Centre that could house a dedicated MIRERC Secretariat, and a supportive University Management Board.

The weaknesses include lack of a graduate school to assure scientific rigor of proposals before submission to the MIRERC, lack of research ethics training in most school's curricula, absence of a dedicated MIRERC Secretariat, undergraduate research proposals being not ethically reviewed, dearth of faculty trained in research ethics, and lack of an operating budget for MIRERC work.

The opportunities include existence of about 22 accredited IRERCs in Kenya, existence of international standards and operational guidance for ethics review, availability of guidelines and codes of best ethical practice in research, existence of a free automated platform called RHInnO Ethics for managing the ethics review process, and availability of external resources for strengthening IRERCs.

In order to improve the performance and sustainability of the MUST research ethics system, it is important to include research ethics training in all undergraduate and post-graduate curricula, create a dynamic database of potential research ethics reviewers, allocate a percentage of the annual MUST research budget for MIRERC operations, charge a graduated fee for proposal ethics review, require all student and faculty internal and external research proposals to be cleared by the MIRERC, and use the RHInnO Ethics platform to manage the ethics review process.

\section{Acknowledgements}

We owe profound gratitude to Jehovah Jireh for meeting all our household needs. J.M.K. is immensely grateful to the MIRERC members, with whom he closely worked with, during his directorship of Research Directorate at the Meru University of Science and Technology, Kenya.

\section{Conflicts of Interest}

The authors declare no conflicts of interest regarding the publication of this paper.

\section{References}

[1] Meru University of Science and Technology (MUST) (2018) Website. http://www.must.ac.ke/ 
[2] MUST (2013) MUST Charter. MUST, Nairobi.

[3] National Council for Law Reporting (2016) Universities Act No. 42 of 2012. Revised Edition 2016. National Council for Law Reporting, Nairobi.

[4] Division of Academic, Research and Student Affairs (2018) Student Enrolment. Division of Academic, Research and Student Affairs, Meru.

[5] Commission for University Education (CUE) (2014) Universities Standards and Guidelines. CUE, Nairobi.

[6] National Council for Science and Technology (NCST) (2009) Procedures and Guidelines for Research Authorization in Kenya. National Council for Science and Technology, Ministry of Higher Education, Science and Technology, Nairobi.

[7] NCST (2004) Guidelines for Ethical Conduct of Biomedical Research Involving Human Subjects in Kenya. NCST, Ministry of Higher Education, Science and Technology, Nairobi.

[8] MUST (2017) Standard Operating Procedures of the MUST Institutional Research Ethics Review Committee (MIRERC). MUST, Meru.

[9] Council for International Organizations of Medical Sciences (CIOMS) (2016) International Ethical Guidelines for Health-Related Research Involving Humans. CIOMS, Geneva.

[10] The World Medical Association (2015) Medical Ethics Manual. World Medical Association, Ferney-Voltaire Cedex.

[11] American Psychological Association (APA) (2012) Guidelines for Ethical Conduct in the Care and Use of Nonhuman Animals in Research. APA, Washington DC.

[12] Council of Europe (2012) Guide for Research Ethics Committee Members. Steering Committee on Bioethics, Brussels.

[13] World Health Organization (WHO) (2009) Research Ethics Committees: Basic Concepts for Capacity-Building. WHO, Geneva.

[14] WHO (2009) Operational Guidelines for Ethics Committees That Review Biomedical Research. Document TDR/PRD/ETHICS/2000.1. WHO, Geneva.

[15] International Council for Harmonisation of Technical Requirements for Pharmaceuticals for Human Use (ICH) (1997) Good Clinical Practice. ICH, Geneva.

[16] National Commission for the Protection of Human Subjects of Biomedical and Behavioural Research (1979) The Belmont Report Ethical Principles and Guidelines for the Protection of Human Subjects of Research. The National Commission for the Protection of Human Subjects of Biomedical and Behavioural Research, Washington DC.

[17] World Medical Association (2013) Declaration of Helsinki: Ethical Principles for Medical Research Involving Human Subjects. JAMA, 310, 2191-2194.

https://doi.org/10.1001/jama.2013.281053

[18] U.S. Government Printing Office (1949) Trials of War Criminals before the Nuremberg Military Tribunals under Control Council Law No. 10, Vol. 2, pp. 181-182. Nuremberg, October 1946-April 1949. U.S. Government Printing Office, Washington DC.

[19] Vargesson, N. (2015) Thalidomide-Induced Teratogenesis: History and Mechanisms. Birth Defects Research, 105, 140-156. https://doi.org/10.1002/bdrc.21096

[20] Reverby, S.M. (2009) Examining Tuskegee: The Infamous Syphilis Study and Its Legacy. University of North Carolina Press, Chapel Hill.

[21] Cartwright, S.R. (1988) The Report of the Cervical Cancer Inquiry: The Report of 
the Committee of Inquiry into Allegations Concerning the Treatment of Cervical Cancer at National Women's Hospital and into Other Related Matters. Government Printer, Wellington.

[22] NCST (2011) Guidelines for Accreditation of Ethics Review Committees in Kenya. NCST, Nairobi.

[23] United Nations Development Programme (UNDP) (2016) Human Development Report 2016: Human Development for Everyone. UNDP, New York.

[24] National Council for Law Reporting (2013) Science, Technology and Innovation ACT No. 28 of 2013. Revised Edition 2014, National Council for Law Reporting, Nairobi.

[25] Motari, M., Ota, M.O. and Kirigia, J.M. (2015) Readiness of Ethics Review Systems for a Changing Public Health Landscape in the WHO African Region. BMC Medical Ethics, 16, 82. https://doi.org/10.1186/s12910-015-0078-9

[26] Zielinski, C., Kebede, D., Mbondji, P.E., Sanou, I., Kouvividila, W. and Lusamba-Dikassa, P.S. (2014) Research Ethics Policies and Practices in Health Research Institutions in Sub-Saharan African Countries: Results of a Questionnaire-Based Survey. Journal of the Royal Society of Medicine, 107, 70-76. https://doi.org/10.1177/0141076813517679

[27] National Commission for Science, Technology and Innovation (NACOSTI) (2017) List of Institutional Ethics Review Committees in Kenya. NACOSTI, Nairobi. https://oris.nacosti.go.ke/modules/institutions/accredited_institutions.php

[28] WHO (2011) Standards and Operational Guidance for Ethics Review of Health-Related Research with Human Participants. WHO, Geneva.

[29] WHO (2017) WHO Guidelines on Ethical Issues in Public Health Surveillance. WHO, Geneva.

[30] National Health and Medical Research Council (2013) Australian Code for the Care and Use of Animals for Scientific Purposes. 8th Edition, National Health and Medical Research Council, Canberra.

[31] UNAIDS/WHO (2007) Ethical Considerations in Biomedical HIV Prevention Trials. UNAIDS, Geneva.

[32] UNESCO (2005) UNESCO Universal Declaration on Bioethics and Human Rights. UNESCO, Paris.

[33] Nuffield Council on Bioethics (2002) The Ethics of Research Related to Healthcare in Developing Countries. Nuffield Council on Bioethics, Nuffield.

[34] United Nations (1948) The Universal Declaration of Human Rights. UN, New York.

[35] International Council for Harmonisation (ICH) (2000) Guidelines on Choice of Control Groups and Related Issues in Clinical Trials. ICH, Geneva.

[36] Council of Europe (1997) Convention on Human Rights and Biomedicine. Council of Europe, Brussels.

[37] Council of Europe (2005) Additional Protocol to the Convention on Human Rights and Biomedicine, Concerning Biomedical Research. Council of Europe, Brussels.

[38] Council of Europe (2001) Directive of the European Parliament and of the Council of 4 April 2001 on the Approximation of the Laws, Regulations and Administrative Provisions of the Member States Relating to the Implementation of Good Clinical Practice in the Conduct of Clinical Trials on Medicinal Products for Human Use. Official Journal of the European Communities, 2001, L121/34. Council of Europe, Brussels.

[39] United States Government (2017) United States Food and Drug Administration 
Regulations for the Protection of Human Subjects-Code of Federal Regulations Title 21, Part 50. U.S. Food and Drug Administration, Washington DC.

[40] Mokgatla, B., Bahati, P. and Jsselmuiden, C.I. (2017) Enhancing the Efficiency and Quality of African Research Ethics Review Processes-Through an Automated Review Platform. Journal of AIDS and Clinical Research, 8, 658. https://doi.org/10.4172/2155-6113.1000658

[41] Council on Health Research for Development (COHRED) (2017) RHInnO Ethics Platform. COHRED, Geneva. http://www.rhinno.net/

[42] Makanga, M. (2018) Taking Stock of Progress. EDCTP Newsletter, Vol. 13, 1-6. http://www.edctp.org/web/app/uploads/2018/02/EDCTP-Newsletter_January-2018. pdf

[43] EDCTP (2014) EDCTP Communication Strategy. EDCTP, Cape Town.

[44] John, E. (2005) Fogarty International Center. Review of the Fogarty International Bioethics Education and Career Development Award Program-Final Report. John E. Fogarty International Center, Bethesda.

[45] Bioethics Society of Kenya Website. http://www.bioethicskenya.org/

[46] Wikipedia. List of Academic Databases and Search Engines. https://en.wikipedia.org/wiki/List_of_academic_databases_and_search_engines

[47] Moi University. Service Delivery Charter (2015) Institutional Research and Ethics Committee (IREC). Moi University, Eldoret. 


\section{List of Abbreviations}

APA: American Psychological Association

COHRED: Council on Health Research for Development

CUE: Commission for University Education

DOR: Directorate of Research

DVC-ARS: Deputy Vice-Chancellor Academic, Research and Student Affairs

EDCTP: European and Developing Countries Clinical Trails Partnership

ICF: Informed Consent Form

ICH: International Council for Harmonisation of Technical Requirements for Pharmaceuticals for Human Use

CIOMS: Council for International Organizations of Medical Sciences

IRERC: Institutional Research Ethics Review Committee

JKUAT: Jomo Kenyatta University of Agriculture and Technology

MIRERC: MUST Institutional Research Ethics Review Committee

MRES: MUST Research Ethics System

MSc: Masters of Science

MUST: Meru University of Science and Technology

MRF: MUST Research Fund

NACOSTI: National Commission for Science, Technology and Innovation

NBC: National Bioethics Committee

NCST: National Council for Science and Technology

NRF: Kenya National Research Fund

PhD: Doctor of Philosophy

REC: Ethics Review Committee

RHInnO: Research for Health Innovation Organiser

RPPC: Research, Printing and Publication Committee

SAFS: School of Agriculture and Food Science

SBE: School of Business and Economics

SCI: School of Computing and Information

SDGS: School Departmental Graduate Committee

SEd: School of Education

SEA: School of Engineering and Architecture

SHS: School of Health Sciences

SoN: School of Nursing

SOPs: Standard Operating Procedures

SPAS: School of Pure and Applied Sciences

SWOT: Strengths, Weaknesses, Opportunities, and Threats

UMB: University Management Board

UNESCO: United Nations Educational, Scientific and Cultural Organisation

VC: Vice-Chancellor

WHO: World Health Organization 


\section{Appendix 1: MUST Research Ethics System SWOT Analysis Instrument}

This instrument was used to identify internal and external factors influencing the performance of the MUST research ethics system (MRES).

\footnotetext{
1). What unique knowledge and skills do MRES have?

Strengths

2). What do MUST scholarly community opine that MRES does well?

3). What resources do MRES have?

4). What are the biggest achievements of MRES to date?

5). What could MRES improve?

6). What knowledge and skills are lacking in MRES?

7). In what areas does MIRERC membership need more training in?

Weaknesses

8). What complaints have MIRERC received from its clientele (students, staff, collaborators) about its ethical services?

9). Does MIRERC have a strategic plan and rolling annual action plans? Are those plans comprehensive? Explain.

10). How can MRES convert its weaknesses into opportunities?

11). How can MRES turn its strengths into opportunities?

12). Is there a research ethics related need at MUST that is not being met by MRES? Explain.

13). How is the science and technology terrain changing in Kenya? How can MUST take advantage of those changes to boost performance of MRES?

Opportunities 14). What are the global initiatives, platforms and partnerships that support research ethics system strengthening?
}

15). What opportunities exist nationally for strengthening MRES?

16). What opportunities exist within sub-regional economic communities and within the African Union for strengthening MRES?

17). Is there something that could be improved in the composition of MIRERC membership, e.g. inclusion of lay persons, persons from other universities? Explain.

18). Has MIRERC been accredited by NACOSTI? Explain.

19). What obstacles do MRES face in performing its functions?

20). Could any MRES weaknesses prevent it from attaining its goals?

21). What might cause MRES problems in future? How?

22). Does MRES fully shield MUST from potential legal consequences of unethical research? Explain.

Threats 23). Is all research MUST students (undergraduate, post-graduate), staff and collaborators are involved in cleared by MIRERC? Explain.

24). Are all MIRERC members knowledgeable in the key international research ethics standards and guidelines? Explain.

25). Do MIRERC members have conflict of interest that may bias their decisions and recommendations? Explain.

26). Are all students and academic / research staff in MUST trained in research ethics, MIRERC standard operating procedures and its tools? Explain.

Source: Developed by authors. 